\title{
Consumo de comida taiwanesa como forma de aculturación: caso Taiwán-Santa Tecla
}

Paola Navarrete Gálvez

Recibido: 10/09/2013 - Aceptado: 16/09/2013

\begin{abstract}
Resumen
Este artículo es producto de la tesis de investigación "Presencia de rasgos culturales taiwaneses en la vida cotidiana de Santa Tecla", para optar a la Licenciatura en Antropología, en la cual se midieron diferentes elementos o rasgos de la cultura taiwanesa en Santa Tecla, El Salvador. Haciendo énfasis en uno de los rasgos culturales identificados: el consumo de comida taiwanesa; y de qué manera ayuda a culturalizar a los individuos de una sociedad; cómo la comida propicia la difusión cultural y, a su vez, permite que la comunidad taiwanesa se inserte y adapte a la sociedad. El estudio es de carácter multimodal, realizándose un estudio descriptivo de poblaciones tanto mediante encuestas como, a su vez, estudios de casos múltiples
\end{abstract}

\section{Palabras clave}

Aculturación, antropología social, extranjeros, gastronomía.

\begin{abstract}
This article is part of the research thesis to qualify for the degree in Anthropology, called "Presence of Taiwanese cultural traits in the daily life of Santa Tecla", which measured different elements or traits of Taiwanese culture in Santa Tecla, El Salvador. This article emphasizes on one of the cultural features identified, the consumption of Taiwanese food by the community, and how it helps to culturalize individuals in the society as food promotes cultural diffusion and in turn allows Taiwanese community to insert and adapt to society. The study is of multimodal character, in which it was conducted a descriptive study of populations through surveys, and multiple case studies.
\end{abstract}

\section{Keywords}

Acculturation, social anthropology, foreigners, gastronomy, immigrants in El Salvador.

\section{Introducción}

Las sociedades humanas han estado desde tiempos remotos en constante migración y movimiento, lo que le ha permitido, al entrar inevitablemente en contacto con otros grupos culturales, la difusión y el traspaso de diversos elementos culturales. Al establecernos y habitar en ciudades, estas se fueron componiendo por diversos grupos con orígenes culturales distintos en su totalidad, que también forman parte de la identidad de la ciudad. Actualmente, El Salvador no es ajeno a este fenómeno; y si bien recibe en sus ciudades pocos grupos de migrantes extranjeros, estos se encuentran latentes dentro de la ciudad.

Según datos del Ministerio de Justicia y Seguridad Pública [MJSP] (2011), una de las comunidades con mayor número de residentes registrados en el país es la taiwanesa, la más numerosa proveniente del continente asiático; y desde el año 2006 hasta junio del 2011, la que tiene más altos índices de naturalización (15, $1 \%$ ), con veintitrés casos registrado. Siendo, además, El Salvador uno de los pocos países del mundo en entablar relaciones diplomáticas con 
Taiwán, lo que ha permitido la transferencia de tecnología, intercambios estudiantiles, becas educativas, adopción de consumo de productos y servicios de origen taiwanés, entre otros. A pesar de esto, y de la existencia de una relación tanto política como cultural en nuestro país, no se ve una presencia activa de esta etnia. A inicios del año 2012, la Asamblea Legislativa decretó el 22 de febrero como el "Día de la Etnia China en El Salvador" (Ochoa, 2012), incluyendo tanto a ciudadanos de la República Popular de China o China Continental como de la República de China o Taiwán. Este decreto pone en evidencia el aumento de las comunidades chinas en El Salvador y su necesidad de ser reconocidos por parte de la nación salvadoreña.

El estudio se centró en el municipio de Santa Tecla, como cabecera del departamento de la Libertad, que es uno de los que tiene mayor porcentaje de población extrajera $(0,89$ \%) entre su población (Dirección General de Estadística y Censos [Digestyc], 2009), un poco mayor que en San Salvador $(0,77 \%)$.

Esta investigación es de carácter multimodal y plantea la utilización de instrumentos estadísticos dentro de la antropología, así como instrumentos cualitativos, permitiendo identificar los rasgos culturales más significativos mediante los cuales la población tecleña interactúa con la población taiwanesa. En este caso, el consumo de comida asiática resultó ser un indicador para establecer que existe presencia y contacto entre los tecleños y la cultura taiwanesa, como además servir como un elemento de adaptación cultural que ha permitido la integración de ambas culturas.

\section{Método}

\section{Diseño y tipo de estudio}

El estudio es de carácter multimodal, descriptivo de poblaciones mediante encuestas con muestras probabilísticas, como a su vez un estudio de caso de carácter múltiple (Montero \& León, 2007).

\section{Participantes del estudio}

Personas de género masculino y femenino, mayores de 13 años, con nacionalidad salvadoreña, residentes en el área de Santa Tecla. Así mismo, miembros de la comunidad chinataiwanesa que tuvieran algún contacto con el municipio de Santa Tecla.

\section{Universo y muestra}

El universo del estudio fue la población tecleña, estimándose una muestra total de 306 participantes, de una población total de 121.908, en base a la fórmula para el cálculo de poblaciones finitas, con un nivel de confianza del $92 \%$ y un margen de error del $5 \%$. Las encuestas se pasaron mediante muestreo al azar.

\section{Instrumentos de recolección de datos}

Se utilizaron dos instrumentos: encuestas con distintas escalas, entre ellas dicotómicas; y de Likert con cuatro niveles, siendo estos: 1. Nada, 2. Poco, 3. Bastante y 4. Mucho, con un número total de 51 ítems. Al realizarse la validación del instrumento se midieron cinco factores, con un valor alfa de la escala total de 0.877 . Entre sus factores se obtuvo un valor alfa de 0.716 para Consumo mediático, 0.768 en Participación en eventos o actividades culturales, 0.795 con las Prácticas de consumo de alimentos taiwaneses, 0.447 en Aprender sobre otra cultura y 0.806 con Compra artículos extranjeros. Al realizar el análisis factorial para los cinco factores y aplicarse la prueba $\mathrm{KMO}$, se obtuvo un valor de 0.777, indicando la adecuación de los datos y el grado de correlación entre ellas como positivo, complementándose con una prueba de esfericidad de Bartlett $(x 2=702.928 ; p$ $=0.000$ ). El análisis se realizó respecto a los cinco factores prefijados, utilizando una rotación de tipo Varimax. Del resultado se obtuvo que los cinco factores expliquen el $67,85 \%$ de la varianza total. Se utilizó una prueba de bondad de ajuste de tipo Kolmogorov-Smirnov, que determinó la utilización de pruebas no paramétricas.

En cuanto al instrumento utilizado para las entrevistas, estas se elaboraron en profundidad de carácter semiestructurado; se realizaron criterios de validez según contenido, constructo y sistematización; y estuvieron basadas en el instrumento realizado por Chen (2008) con población taiwanesa. Los informantes claves se buscaron y seleccionaron según criterios y requisitos establecidos previamente: ser de nacionalidad china-taiwanesa, con dos o más años de residencia, mayor de 18 años de edad, que tuviera algún contacto con el municipio de Santa Tecla, ya sea que viviera o trabajara allí, o formara parte y participara dentro de una comunidad de esa localidad, como que hablara suficiente español para entender y entablar una conversación sin dificultad (clasificación EU A2-B1). 


\section{Resultados}

Tabla 1.Características sociodemográfica de los participantes

\begin{tabular}{|c|c|c|}
\hline Características & $\mathbf{F}$ & $\%$ \\
\hline \multicolumn{3}{|l|}{ Sexo: } \\
\hline Masculino & 206 & 70,5 \\
\hline Femenino & 86 & 29,5 \\
\hline \multicolumn{3}{|l|}{ Sector trabajo: } \\
\hline Formal & 125 & 42,1 \\
\hline Informal & 34 & 11,4 \\
\hline Subempleo & 2 & 0,7 \\
\hline No trabaja & 136 & 45,8 \\
\hline \multicolumn{3}{|l|}{ Nivel educativo completado: } \\
\hline Primaria & 38 & 12,9 \\
\hline Secundaria & 62 & 21,1 \\
\hline Media & 140 & 47,6 \\
\hline Superior & 50 & 17,0 \\
\hline
\end{tabular}

\section{Frecuencias generales de la encuesta}

Entre los resultado obtenidos se tiene que el $22,5 \%$ de la población tecleña aseguró haber probado comida taiwanesa; un $66,1 \%$ nunca la había probado, y un $11,4 \%$ dijo no saber si la había probado o no. Estos datos están acordes con lo esperado debido a que se está midiendo una cultura extranjera dentro de la local, mostrando una frecuencia de consumo muy poca $(\mathrm{M}=1.38$; $\mathrm{DT}=0.603)$.

Tabla 2. Conocimiento de platillos Taiwaneses según nivel educativo

\begin{tabular}{lcccc}
\multicolumn{1}{c}{ Preguntas } & Secundaria (\%) & Media (\%) & Superior (\%) & Total (\%) \\
\hline Bubble Tea & $\mathbf{2 9 , 6}$ & 15,8 & 13,7 & 18,3 \\
Galletas rellenas chinas & $\mathbf{7 1 , 9}$ & 46,4 & 43,4 & 49,8 \\
Bollos al vapor & $\mathbf{4 0 , 7}$ & 28,6 & 34,9 & 35,9 \\
Wantanes & 33,3 & 32,1 & $\mathbf{4 1 , 7}$ & $\mathbf{4 4 , 7}$ \\
\hline
\end{tabular}

Al preguntar por platillos específicos, muchas más personas dijeron haber probado esta comida, siendo unos de los platillos más reconocidos o probado la galleta rellena china, con un total del $49,8 \%$ de la población total; seguido por los wantanes, con un $44,7 \%$. Entre la población que dijo haber probado o conocer las galletas, el porcentaje más alto, con $71,9 \%$, eran personas que estaban estudiando 0 habían estudiado hasta la educación secundaria. En el caso de wantanes, el 41,7 \% manifestó haber estudiado hasta la educación superior. Se esperaba que se conociera más el platillo de wantanes debido a su familiaridad no solo taiwanesa, sino además en restaurantes chinos; pero es posible que las galletas tengan un porcentaje mayor debido a su dimensión cotidiana. Estas galletas se pueden comprar rápidamente en la calle debido a que usualmente no es necesario entrar al restaurante para comprarlas, a diferencia 
de los wantanes que generalmente son consumidos adentro de los restaurantes. Otro factor que podría incidir es la opinión que los precios de los platillos dentro de los restaurantes de comida taiwanesa no son considerados accesibles (M = 1.42; DT = 0.637), y las personas optarían por consumir productos con precios más bajos. En este caso, la preferencia a consumir las galletas. También se preguntó en qué áreas de su vida cotidiana estaban presentes o había mayor presencia asiática. El área que demostró mayor presencia asiática, aunque poca, fue en la categoría Centro comercial, con un $34,3 \%$ que consideró que era cotidiano verlos; dentro de la misma área, un 37,9 \% lo consideró que era común verlos, al igual que era común en los parques $(34,9 \%$ ) y el mercado $(35,5 \%)$. Tentativamente, se podría opinar que es posible verlos más en comercios, tiendas y restaurantes que en otros lugares de la ciudad, como en el área residencial, las universidades, los colegios, parques, etc.

\section{Factor Consumo de comida según variables sociodemográficas}

Al analizar el factor Consumo de alimentos, mediante la prueba de Kruskall-Wallis con la variable edad, resultó que la edad es determinante para su aparición (X2 = 83.967, Sig $=0.000$ ).

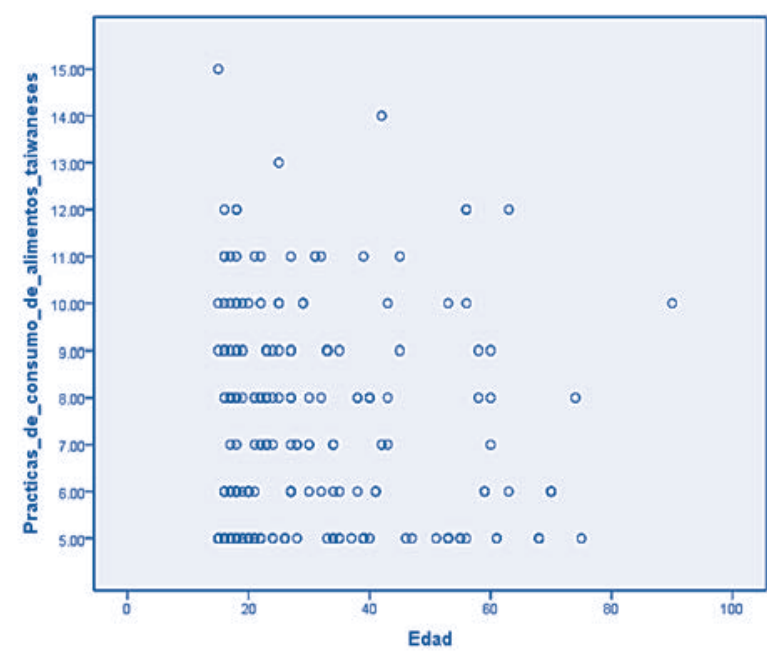

Figura 1.

Factor Prácticas de consumo de alimentos distribuido por edad

En la figura 1, el rango de edad se concentra entre 15 a 30 años. Con un rango de 5 a 10 de prácticas de consumo, también nos indica un consumo de alimentos taiwaneses de poco a moderado.

Al hacer la prueba con la variable de trabajo, mostró significancia $(\mathrm{X} 2=8.124, \mathrm{p}=0.043)$, posiblemente en cuanto a que disponer de un trabajo facilita el consumo de alimentos extranjero en restaurantes.

Tabla 3. Correlaciones entre factores estudiados

\begin{tabular}{|c|c|c|c|c|c|}
\hline & $\begin{array}{l}\text { Consumo } \\
\text { mediático }\end{array}$ & $\begin{array}{c}\text { Participación } \\
\text { eventos o } \\
\text { actividades } \\
\text { culturales }\end{array}$ & $\begin{array}{c}\text { Prácticas de } \\
\text { consumo de } \\
\text { alimentos } \\
\text { taiwaneses }\end{array}$ & $\begin{array}{c}\text { Aprender } \\
\text { sobre otras } \\
\text { culturas }\end{array}$ & $\begin{array}{l}\text { Comprar } \\
\text { artículos } \\
\text { extranjeros }\end{array}$ \\
\hline \multirow{2}{*}{ Consumo mediático } & & .369 & $.508^{*}$ & .340 & .422 \\
\hline & & .000 & .000 & .000 & .000 \\
\hline \multirow{2}{*}{$\begin{array}{l}\text { Participación eventos } 0 \\
\text { actividades culturales }\end{array}$} & .369 & & .398 & .169 & .210 \\
\hline & .000 & & .000 & .005 & .001 \\
\hline \multirow{2}{*}{$\begin{array}{l}\text { Prácticas de consumo de } \\
\text { alimentos taiwaneses }\end{array}$} & .508 & .398 & & .124 & .510 \\
\hline & .000 & .000 & & .056 & .000 \\
\hline \multirow{2}{*}{ Aprender sobre otras cultura } & .340 & .169 & .124 & & .256 \\
\hline & .000 & .005 & .056 & & .000 \\
\hline \multirow{2}{*}{ Comprar artículos extranjeros } & .422 & .210 & .510 & .256 & \\
\hline & .000 & .000 & .000 & .000 & \\
\hline
\end{tabular}

*Las correlaciones son significativas a $p<0.01$. 
Entre los factores Consumo mediático y Prácticas de consumo de alimentos taiwaneses se encontró correlación de $r=0.508$, como además una relación entre Comprar artículos extranjeros y Prácticas de consumo de alimentos taiwaneses $(r=0.510)$. Para determinar la linealidad de estas correlaciones detectadas, se realizaron gráficos de regresión lineal. Las regresiones nos permiten, además, predecir valores entre las variables dependientes sobre una o más variables independientes (Hernández, Fernández y Baptista, 2006).

Así, tenemos que entre la correlación Consumo Mediático y Prácticas de consumo de alimentos taiwaneses $(r=0.508)$, siendo la variable dependiente las prácticas de consumo de alimentos que mostraron un valor de $\mathrm{R}^{2}$ de 0.25 , indicando que el $25 \%$ puede ser explicado, lo cual sugiere que en 1 de cada 4 casos el consumo mediático habrá condicionado el consumo de alimentos. Siendo el consumo mediático mediante la prueba $t$ variables significativas para las prácticas de consumo de alimentos $(p=0.000)$.

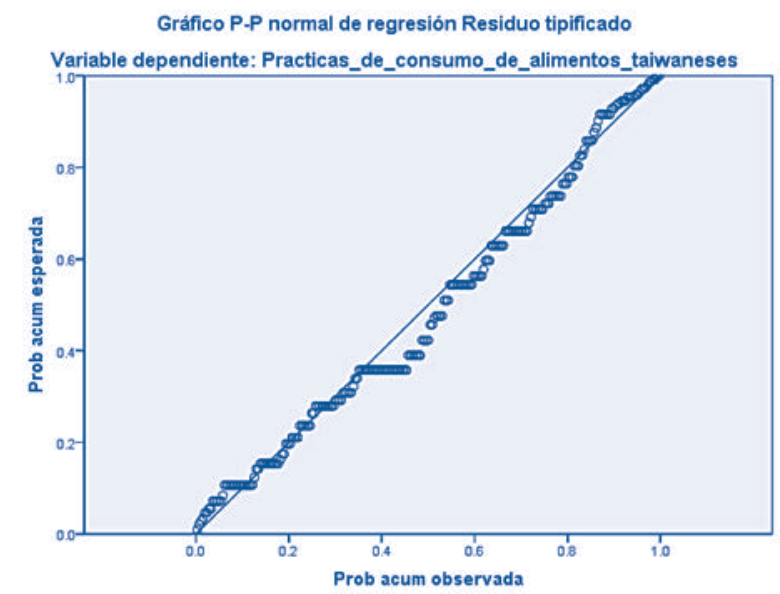

Figura 2.

Gráfico P-P normal de regresión residuo tipificado de consumo mediático y prácticas de consumo de alimentos taiwaneses

La dinámica entre ambos factores muestra una tendencia lineal, similar a la línea recta dentro del modelo, donde la probabilidad esperada es muy similar a la probabilidad explicada por el modelo. Pero siempre indicando un bajo consumo mediático y prácticas de consumo de alimentos taiwaneses por parte de los encuestados.

Para los factores Compra artículos extranjeros y Prácticas de consumo de alimentos taiwaneses, siendo esta la variable dependiente, en donde el valor $\mathrm{R}^{2}(0.260)$ independiente explica únicamente un $26 \%$ de la variable dependiente, de la misma forma que la relación anterior, predice 1 de 4 casos con certeza. Teniendo los resultados de la prueba t una significación de $p=0.000$, se puede tomar el modelo como válido. La dinámica muestra una tendencia lineal, similar a la línea recta dentro del modelo, aunque la probabilidad observada difiere de mayor manera a la probabilidad esperada.

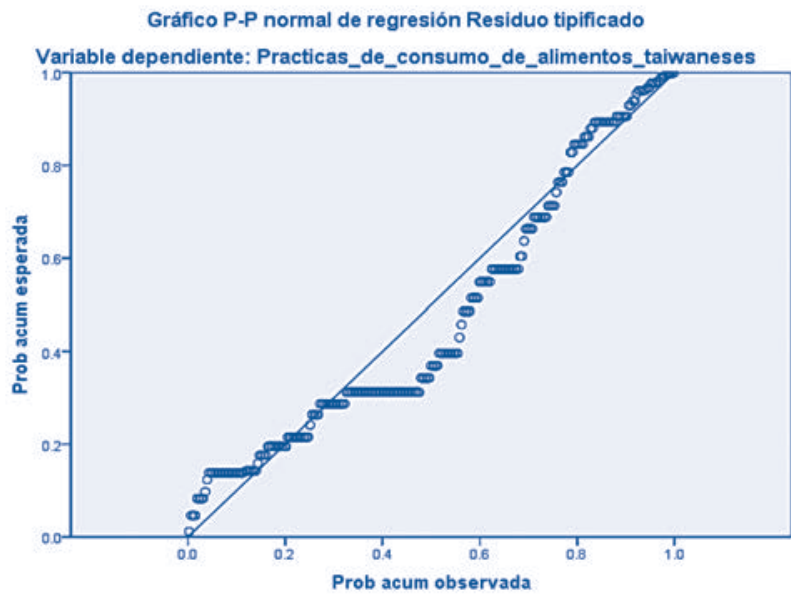

Figura 3.

Gráfico P-P normal de regresión residuo tipificado de compra artículos extranjeros y prácticas de consumo de alimentos taiwaneses

Pero también se indica una cantidad mediana de compra de artículos extranjeros y prácticas de consumo de alimentos taiwaneses. Con estos datos se podría predecir que entre mayor consumo mediático asiático, hay una mayor práctica de consumo de alimentos, al igual que entre más alto sea el nivel de compra de artículos extranjeros, habrá un mayor consumo de alimentos taiwaneses.

\section{Resultados del instrumento cualitativo}

El estudio abordó los dos ámbitos principales de los procesos de aculturación y difusión intercultural: la cotidianidad de Santa Tecla, por medio de las encuestas; y la comunidad taiwanesa, cuya cultura está afianzada entre sus miembros, transmitiéndose así a la comunidad, para lo cual se utilizaron las entrevistas.

Se realizaron un total de ocho entrevistas a miembros de distintas familias taiwanesas, tomando a cada uno como 
casos únicos, en distintos papeles sociales dentro de su comunidad y la salvadoreña, por ejemplo, como madre de familia, estudiante universitario, empresario, etc. Para analizar el contenido de las entrevistas, se codificaron todas las transcripciones, como también las anotaciones sobre Ios entrevistados. Las codificaciones se realizaron teniendo en cuenta los objetivos, enfocándose en elementos que denotaran en qué áreas tenían mayor presencia y manifestaban cierta influencia hacía su comunidad cercana; elementos que no estuvieran en estas categorías fueron excluidos. Una vez codificadas, se realizó un análisis de conglomerado, agrupándolas según los nodos establecidos, tomando de base la similitud de las palabras empleadas. A partir de esto, se realizó un análisis del coeficiente de correlación de Pearson para determinar la relación entre los nodos.

Una de las correlaciones más fuertes fue la relación entre los nodos Presencia e influencia de restaurantes y restaurantes taiwaneses.

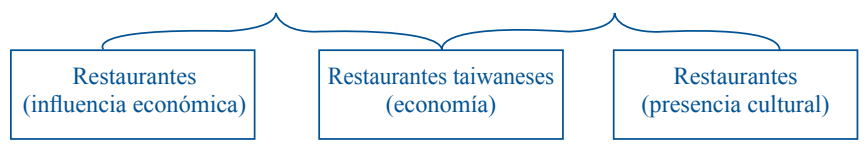

Figura 4.

Relación entre los nodos presencia e influencia de restaurantes y restaurantes taiwaneses

El nodo Restaurantes taiwaneses como presencia cultural se vio relacionado con Restaurantes como presencia económica y Restaurantes como forma de influencia económica, ambos con una correlación de 0.625 , indicando que la presencia cultural de los restaurantes taiwaneses va ligada fuertemente a los factores económicos. Varios de estos restaurantes han sido creados como formas de subsistencia de estas familias, y como consecuencia, indirectamente, han logrado mediante ellos transmitir parte de su cultura, con platillos taiwaneses, creencias (al tener restaurantes vegetarianos) y creando un espacio en el cual logran interactuar con la población local.

\section{Discusión}

\section{Comida taiwanesa}

El estudio abordó los dos ámbitos principales de los procesos de aculturación, entendiendo a esta como el intercambio cultural que se realiza al encontrarse dos culturas distintas, y la difusión intercultural. Al comparar ambos grupos culturales bajo el enfoque de Berry $(1997,2005,2011)$, el proceso de aculturación entre la comunidad taiwanesa y la salvadoreña ha sido de dos vías. Ambas están en contacto e intercambian información, con una diferencia de escala en cuanto a nivel de asimilación de rasgos culturales, conductas y modos de vida, siendo la comunidad taiwanesa la que más rasgos debe asimilar.

La aculturación entre la comunidad salvadoreña y la taiwanesa se da de forma espontánea (Cuche, 1999), surgiendo entre la interacción de ambas y no de manera forzada. En el caso del consumo de alimentos taiwaneses en restaurantes, este mismo elemento constituye un proceso que contribuye a la difusión cultural por medio de la oferta de comida de dicha etnia. Siendo esto una manera de aculturación por sí misma, ya que, como lo manifiesta Cuche (1999), los elementos materiales se transmiten con mayor facilidad que los culturales simbólicos. Al ser el proceso de aculturación bilateral, no solamente los tecleños conocen más de esta cultura y consumen sus platillos. Varias de sus recetas han tenido que ser modificadas para adaptarse al gusto local, manifestando la aculturación de ambas vías. Tradicionalmente, esta galleta se rellena de frijol dulce, pero aquí ha adaptado distintas variedades de sabores, entre ellas chocolate, guineo, vainilla y café, debido a la repulsión entre los salvadoreños de consumir frijoles dulces, porque tradicionalmente los frijoles se consumen salados, y por eso causaba entre los que probaban dichas galletas cierta aversión al comerlas. Incluso se intenta traducir los platillos al leguaje local, como llamarle galleta china, cupín o pancitos chinos.

Esto también es explicado por una de las leyes planteadas por Bastide (Pereira, 2007) en donde menciona que entre más extraña sea la forma de un rasgo cultural, más dificultosa será la recepción.

\section{Restaurantes taiwaneses}

Los restaurantes, además, manifestaron ser un espacio en donde era posible interactuar de manera natural y permitir, sin mucho esfuerzo, un intercambio cultural. No solo es el restaurante un medio de transmisión de su cultura a la comunidad salvadoreña, sino que, además, tecleños interesados en probar esta comida también estaban interesados en saber más sobre su cultura. Todos los entrevistados que poseían un restaurante de comida taiwanesa manifestaron que, además, debido a la solicitud de sus clientes o paralelamente a su trabajo, daban clases de mandarín, usualmente impartidas en el mismo local. A veces dos generaciones en la familia venían dando clases 
de mandarín a salvadoreños en sus establecimientos, convirtiéndose en lugares no solo de transmisión física de elementos culturales, sino en espacios que propician la transmisión de creencias, cosmovisiones y lenguaje.

Samantha Barbas (2003) hace notar la capacidad que tienen los restaurantes de lograr una interacción entre culturas y una diversificación en la culinaria local, manifestando que en muchas ocasiones estos establecimientos son más exitosos en promover el intercambio entre diversas culturas y tradiciones que las instituciones sociales.

Si bien, los restaurantes permiten un contacto entre los dueños del restaurante y las personas que llegan a consumir, no sucede así si otros taiwaneses llegasen a consumir en el restaurante. Se descubrieron dos tipos de interacción entre la comunidad taiwanesa con la salvadoreña.

Basandose en Berry $(1997,2005,2011)$, se han encontrado dinámicas tanto de separación como de integración, entendiendo separación como darle un mayor valor a mantener su cultura original, y, al mismo tiempo, desear evitar interaccionar con otros miembros distintos a su grupo, que principalmente se da entre los individuos migrantes de primera generación taiwanesa, entre cuyos miembros, especialmente los de mayor edad, existen los que incluso se niegan a hablar otra lengua que no sea el chino mandarín; y la dinámica de integración, la cual, aunque casi exclusiva de los miembros de segunda generación de la comunidad, también se da entre los miembros más jóvenes de la primera, en especial aquellos relacionados al ámbito económico y el educativo, lo que sugiere que dicha integración se da forzosamente (Cuche, 1999) por las relaciones sociales que el vivir en sociedad requiere. Los miembros de segunda generación, entre niños y jóvenes, manifiestan facilidad para hablar el idioma y la integración con sus pares locales; mas existen diferencias específicas respecto a la dieta.

\section{Conclusiones}

Uno de los rasgos culturales más sobresalientes de la cultura taiwanesa presente en el municipio de Santa Tecla ha sido el factor Prácticas de consumo de alimentos taiwaneses. Se recolectaron insumos que sugieren que de parte de los jóvenes tecleños existe un mayor interés y apertura hacia probar y consumir comida de culturas distintas o exóticas, en este caso la taiwanesa. Siendo además la población del sector laboral la que manifestó hacer un mayor consumo de alimentos taiwaneses, en comparación a la población que no trabaja o que está subempleada. Tomando en cuenta que la percepción que tienen los clientes es que el precio de la comida taiwanesa es alto.

Entre la población encuestada, se encontró una relación entre el consumo mediático con las prácticas de consumo de comida taiwanesa. Esta relación muestra que las personas que consumen productos mediáticos, ya sea que vean novelas o programas documentales de origen asiático, tienen estadísticamente mayores posibilidades de probar o consumir comida taiwanesa.

Los restaurantes como espacio físico, además, resultaron ser lugares de intercambio cultural no solo por el hecho de que transmiten parte de su cultura mediante la gastronomía taiwanesa, sino, además, porque en los restaurantes se mantienen decoraciones, imágenes, simbologías e iconos que manifiestan su cultura y sus creencias. Y como además en los restaurantes están los dueños presentes, esto permite una interacción directa para el intercambio cultural con los comensales.

Si bien no existe un alto número de taiwaneses habitando en Santa Tecla, los pocos presentes han logrado adaptarse a su entorno inmediato modificando su propia cultura y la de los salvadoreños. A pesar de que todavía una minoría de la población local ha visitado o conoce los restaurantes o la comida taiwanesa, se hace notar la importancia que tendrían estos elementos como un medio de intercambio e interacción cultural. 


\section{Referencias}

Barbas, S. (2003). «|»\| Take Chop Suey': Restaurants as Agents of Culinary and Cultural Change. The Journal of Popular Culture, 36(4), 669-686. doi:10.1111/15405931.00040.

Berry, J.W. (1997). Immigration, acculturation, and adaptation. Applied psychology: An international review, (46 (I)), pp. 5- 68.

Berry, J.W. (2005). Acculturation: Living successfully in two cultures. International Journal of Intercultural Relations, 29(6), pp. 697-712. doi:10.1016/j.ijintrel.2005.07.013.

Berry, J.W. (2011). Integration and multiculturalism: Ways towards social solidarity. Peer Reviewed Online Journal, Vol. 20, pp. 2.1-2.21.

Chen, C. (2008). Getting saved in America: Taiwanese Immigration and Religious Experience. Estados Unidos: Princeton University Press.

Cuche, D. (1999). La noción de cultura en las ciencias sociales. Buenos Aires: Nueva Visión.

Ministerio de Justicia y Seguridad Pública [MJSP]. (2010, febrero 12). Proceso de Salida e Ingreso de El Salvador. Dirección General de Migración y Extranjería.
Recuperado 23 de abril de 2012, a partir de http:// www.seguridad.gob.sv/index.php?option=com content\&view $=$ article\&id $=257$

Ministerio de Justicia y Seguridad Pública (MJSP). (2011, abril 15). Estadísticas de migración. Dirección General de Migración y Extranjería. Recuperado: 19 de abril de 2012, a partir de http://www.seguridad.gob.sv/ index.php?option=c om_content\&view=article\&id =98:estadisticas-de-migracion\&catid=35:todos-losservicios\&ltemid $=172$

Montero, I. \& León, O. G. (2007). A guide for naming research studies in psychology. International Journal of Clinical and Health Psychology, Vol. 7(No. 3), pp. 874-862.

Ochoa, I. (2012, enero). El «Día de la etnia china en El Salvador» será celebrado cada 22 de febrero. Asamblea Legislativa República de El Salvador. Recuperado a partir de http://asamblea.gob.sv/noticias/archivode-noticias/el-201cdia-de-la-etnia-china-en-elsal vador201d-sera-celebrado-cada-22-de-febrero.

Pereira, L. (2007). Roger Bastide y los límites de la antropología aplicada tradicional. Boletín Antropológico, 25 (69), pp. 29-56. 